\title{
Serum bilirubin studies in patients with intermittent intrahepatic cholestasis
}

\author{
R. BRODERSEN AND N. TYGSTRUP \\ From the Department of Biochemistry A, University of Copenhagen, \\ and Medical Department $A$ and B, Rigshospitalet, Copenhagen, Denmark
}

EDITORIAL COMMENT Rapid elimination of injected bilirubin with low serum concentrations of unconjugated bilirubin indicates a hyperactive conjugation mechanism. The cholestatic syndrome may be due to an excess of alkali-stable monoconjugate.

The syndrome of intermittent intrahepatic cholestasis of unknown aetiology has been described by several authors, and Summerskill (1965) has surveyed the cases reported recently. The present work, mainly based on specific methods for the determination of unconjugated bilirubin and bilirubin diglucuronide, indicates that an abnormality in the metabolism of bilirubin may play a role in this syndrome, at least regarding the type seen in young males from the Faroe Islands.

Of the five patients included in this study, two have been presented in a previous report (Tygstrup, 1960). The clinical features are essentially the same in all the patients. The diagnosis is based on the following findings: 1 Intermittent jaundice with pruritus, the attacks usually lasting several months, and occur with regular or irregular intervals. Between the attacks the patients are symptom-free. 2 The liver tests are suggestive of cholestasis, i.e., mainly direct reacting bilirubin, high alkaline phosphatases, and moderately elevated transaminases. 3 Liver biopsy shows bile plugs and no signs of hepatitis or cirrhosis. 4 Per-operative cholangiography shows patent intra- and extrahepatic bile ducts.

In three patients the syndrome appeared during childhood, in the remaining two (nos. 2 and 5) about the age of 20 .

\section{METHODS}

Unconjugated bilirubin in serum was determined by chloroform extraction (Brodersen and Vind, 1963). Total serum bilirubin was estimated by the method of Powell (1944), slightly modified (Brodersen, Hermann, and Vind, 1963). Bilirubin diglucuronide in serum was identified and measured bj a radio-isotope derivative method (Brodersen, 1966).

In the serum from one patient the kinetics of coupling with diazobenzenesulphonic acid was investigated. Coupling was done in an aqueous medium at $p \mathrm{H} 1.3$ and $730 \mu \mathrm{M}$ diazobenzenesulphonic acid, and also after precipitation of proteins with ethanol ( $92 \%$ by volume) in a medium consisting of chloroform-ethanol-water (3: $6: 1$ by volume) with $0 \cdot 01 \mathrm{M}$ hydrochloric acid and $124 \mu \mathrm{M}$ diazobenzenesulphonic acid. Coupling in both media was also performed after alkaline hydrolysis with $0 \cdot 2 \mathrm{M}$ sodium hydroxide for five minutes. The temperature during hydrolysis and coupling was $25^{\circ} \mathrm{C}$.

Elimination of bilirubin from the blood stream after intravenous injection of $85 \mu$ mole of bilirubin in sterile sodium carbonate solution (Homburg) was investigated by determination of unconjugated bilirubin by the chloroform extraction method.

RESULTS

Table I shows the determination of total bilirubin,

TABLE

CONCENTRATIONS OF BILIRUBIN

\begin{tabular}{|c|c|c|c|c|c|}
\hline $\begin{array}{l}\text { Patient } \\
\text { No. }\end{array}$ & $\begin{array}{l}\text { Sample } \\
\text { No. }\end{array}$ & $\begin{array}{l}\text { Total } \\
\text { Bilirubin } \\
(\mu M)\end{array}$ & $\begin{array}{l}\text { Unconjugated } \\
\text { Bilirubin } \\
(\mu M)\end{array}$ & $\begin{array}{l}\text { Bilirubin } \\
\text { Diglucuronide } \\
(\mu M)\end{array}$ & $\begin{array}{l}\text { Other } \\
\text { Bilirubin } \\
(\mu M)\end{array}$ \\
\hline \multicolumn{6}{|c|}{ Icteric Phase } \\
\hline \multirow[t]{2}{*}{1} & 1 & 271 & 3.8 & 232 & 35 \\
\hline & 2 & 282 & 3.6 & 225 & 53 \\
\hline 2 & 1 & 68 & 4.0 & 35 & 29 \\
\hline 3 & 2 & 133 & 9.0 & 22 & 102 \\
\hline 4 & 2 & 46 & $2 \cdot 4$ & 16 & 27 \\
\hline \multirow[t]{4}{*}{5} & 1 & 18 & $2 \cdot 6$ & & \\
\hline & 2 & 26 & 5.0 & & \\
\hline & 3 & 102 & 3.0 & 62 & 37 \\
\hline \multirow{3}{*}{\multicolumn{2}{|c|}{$\begin{array}{l}\text { Mean }{ }^{1} \\
\text { Anicteric Phase }\end{array}$}} & & $2 \cdot 7$ & & \\
\hline & & & 4.0 & & \\
\hline & & & & & \\
\hline 3 & 1 & $13 \cdot 5$ & $8 \cdot 6$ & $1 \cdot 2^{2}$ & 3.7 \\
\hline 4 & 1 & & $5 \cdot 0$ & & \\
\hline
\end{tabular}

${ }^{1}$ In healthy adult males the average was $6 \cdot 8 \mu \mathrm{M}(2 \cdot 6-17 \cdot 8 \mu \mathrm{M}=95 \%$ limits) with normal distribution of $\log \mathrm{c}$ (Brodersen, Hermann, and Vind, 1963). The difference between mean $\log \mathrm{c}$ of the control subjects and the patients is statistically significant $(p<0.001)$.

${ }^{2}$ In 57 adult males the average was $0.21 \mu \mathrm{M}(0.11-0.40 \mu \mathrm{M}=95 \%$

limits) (Brodersen, 1966). 
unconjugated bilirubin, and bilirubin diglucuronide. Observations made during icteric and anicteric phases are given separately. The difference between total bilirubin and the sum of unconjugated bilirubin and bilirubin diglucuronide is given as 'other bilirubin'.

The samples examined in the 'icteric phase' were taken when the serum bilirubin level was decreasing, except in case 3. Samples 1 and 2 from case 1 , and 1 and 2 and 3 and 4, respectively, from case 5 were taken at an interval of about 10 days. Case 5 was studied during two attacks.

The samples examined in the anicteric phase were taken shortly after total bilirubin had reached the normal level, and serum alkaline phosphatases were still elevated.

An attempt to characterize 'other bilirubin' was made by investigating the kinetics of the diazo

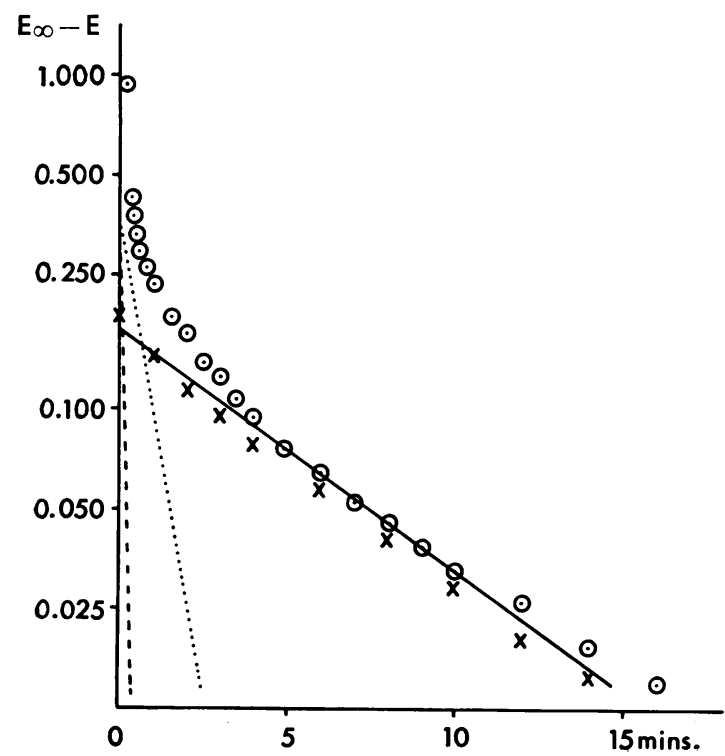

FIG. 1. Kinetics of azo-coupling in native serum in aqueous medium from case 5 . From the curve obtained before alkaline hydrolysis $(\bigcirc \bigcirc \bigcirc \bigcirc \bigcirc)$ three first-order reactions have been calculated, with the following constants:

$1(-----)$ velocity constant $>10^{4}$ min. $^{-1} \mathrm{M}^{-1}$; $E_{\infty}=0.39$

$2(. .$.$) velocity constant 1.8 \times 10^{3}$ min. $^{-1} \mathrm{M}^{-1}$; $E_{\infty}=0.36$

3 (一_-) velocity constant $2.4 \times 10^{2}$ min. $^{-1} \mathrm{M}^{-1}$; $E_{\infty}=0.18$

The data for the two fastest reactions agree with those found for bilirubin diglucuronide (Brodersen, 1966). The curve remaining after alkaline hydrolysis $(\mathrm{xxxx})$ is considered identical with the slowest reaction. coupling (Overbeek, Vink, and Deenstra, 1955). A serum sample from case 5 (sample no. 3 of Table I) was examined under three different conditions. In aqueous solution (corresponding to the direct van den Bergh reaction) the course of the coupling was studied before and after alkaline hydrolysis (Fig. 1). It shows that the serum, in addition to bilirubin diglucuronide, contains an alkali-stable bilirubin conjugate. In the serum sample in question it was found that bilirubin diglucuronide could be removed by precipitation of the proteins by ethanol. In the supernatant from this precipitation it was therefore possible to study the coupling of the unknown pigment in an ethanol-chloroform medium (corresponding to the indirect van der Bergh reaction). From this curve two first order processes could be calculated with practically identical values for $E_{\infty}$. The faster process was twice as fast as the faster phase of coupling of unconjugated bilirubin in this medium, and the rate of the slower process was very close to the rate of the slower process for unconjugated bilirubin. This observation indicates that the serum contains a mono-conjugate of bilirubin.

The elimination of injected bilirubin from the

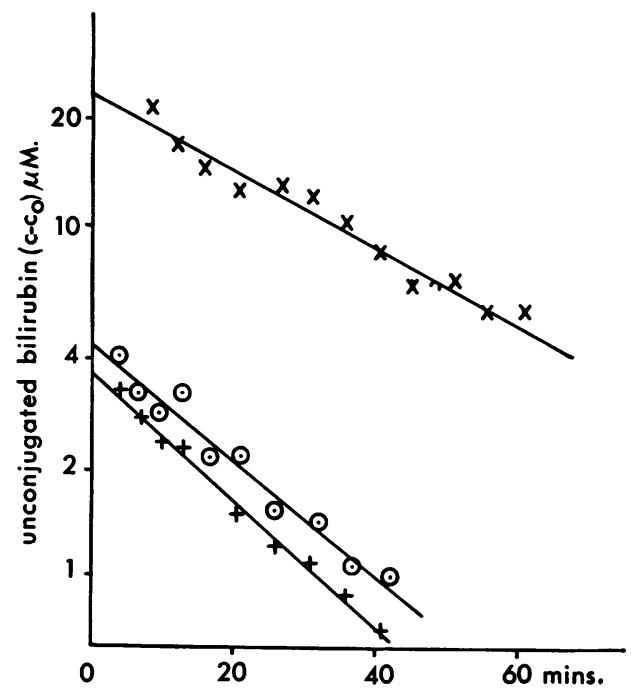

FIG. 2. Elimination of injected bilirubin. From the elimination curves the following constants have been calculated.

Case $4(\bigcirc \bigcirc \bigcirc \bigcirc \bigcirc)$ : elimination constant 0.038 min..$^{-1}$, bilirubin space, 19 litres,

Case $5(+++)$ : elimination constant 0.041 min. $^{-1}$, bilirubin space, 23 litres.

Normal subject $(\times \times \times \times)$ (calculated from the data of Billing et al. (1964): elimination constant 0.025 min..$^{-1}$, bilirubin space, $3 \cdot 7$ litres. 
blood was studied in two patients (cases 4 and 5) during icteric phases. The concentration of unconjugated bilirubin on a logarithmic scale as a function of time is shown in Figure 2. It appears that the elimination with good approximation is a first order reaction. The velocity constants and the distribution volumes, calculated by extrapolation of the curve, are given with the graph. For comparison the corresponding values for a normal person, calculated from the data of Billing, Williams, and Richards (1964), are given. It appears that both the elimination rate and distribution volume of unconjugated bilirubin are increased in the patients.

\section{DISCUSSION}

Cases of constitutional hyperbilirubinaemia can be divided into two main groups: in one the unconjugated and in the other the conjugated bilirubin level is predominantly raised. This is usually determined by the direct and indirect van der Bergh reaction. Further classification by analysis of bilirubin metabolites has not been possible, probably due to lack of specific analytical procedures. The full implications of the present results, which are largely based on specific determination of unconjugated bilirubin and bilirubin diglucuronide, therefore cannot yet be estimated.

It is assumed that the most important finding is the reduced concentration of unconjugated bilirubin in the serum of the patients. This is very unlikely to be a result of cholestasis, but points to a primary abnormality in the metabolism of bilirubin. Bilirubin diglucuronide is readily hydrolyzed to bilirubin during the analytical procedure (Brodersen and Vind, 1963), and considering the high concentration of the diglucuronide in most of the patients examined, one must expect that the actual concentrations of unconjugated bilirubin are even lower than the figures given in Table I. This source of error does not affect the control values, since the concentration of bilirubin conjugates in normal sera is very low (Brodersen, 1966).

The concentration of unconjugated bilirubin apparently is not correlated with total bilirubin or bilirubin diglucuronide. The observations permit no statements on the temporal relationships of the alterations, but the normal values seen in two patients during anicteric phases indicate that the intermittency of the syndrome also concerns the concentration of unconjugated bilirubin.

The nature of the 'other bilirubin' remains obscure. In mixtures of unconjugated bilirubin and bilirubin diglucuronide 'total bilirubin' is a fairly accurate measure of the sum of these substances, and the presence of other pigments therefore in most cases is certain. Their concentrations cannot be calculated from the figures, however, because the molar extinction coefficient of their azo-pigments may be different. In sample no. 3 of case 4 the kinetic studies indicate that an alkali-stable monoconjugate of bilirubin is present, and the data show that this conjugate may account for all or most of the 'other bilirubin'. A monoconjugate with similar properties has been found in normal human bile and in the serum of some healthy subjects (Brodersen, 1962; Brodersen et al., 1963; Brodersen, 1966). The quantity of alkali-stable monoconjugate found in this case greatly exceeds that found in normal sera, but this may not be specific for this type of jaundice.

The elimination of injected bilirubin in the two patients subjected to this procedure was greatly enhanced, in contrast to the findings of Williams, Cartter, Sherlock, Scheuer, and Hill (1964) in one patient suffering from a similar syndrome. Methodological causes for the discrepancy cannot be excluded, however. The most striking finding is the large apparent volume of distribution. The true distribution volume of bilirubin is not known, but the data of Billing et al. (1964) indicate that in normal man in short-term experiments it approximates the intravascular volume. If this also applies to our patients it must mean that about $80 \%$ of the amount injected has been removed during the first three minutes. This again makes it probable that a considerable part of the bilirubin has been eliminated or conjugated outside the liver.

The rapid elimination of injected bilirubin and the low concentrations of unconjugated bilirubin in serum can be explained by a hyperactive conjugation mechanism in these patients. Whether formation of an abnormal conjugate, for instance an alkali-stable monoconjugate, is responsible for the cholestatic syndrome which is the predominant clinical feature of these patients, remains to be elucidated.

\section{SUMMARY}

In five young Faroe male patients with intermittent intrahepatic cholestasis of unknown aetiology the serum level of unconjugated bilirubin during icteric phases was found to be lower than in normal subjects. The concentration of bilirubin diglucuronide was elevated, but not sufficiently to account for all the bile pigment in the serum. Specific analytical procedures were used for determinations of unconjugated bilirubin and bilirubin diglucuronide. Reaction kinetic studies performed on a blood sample from one of the patients indicated the presence of an alkali-stable monoconjugate of bili- 
rubin. These findings, together with an abnormally rapid disappearance of injected unconjugated bilirubin in two of the patients, can be explained by a hyperactive conjugation mechanism.

We wish to thank Dr. Bendt Jensen, Physician-in-Chief, Landssjukrahusid, Torshavn, the Faroe Islands, for referring the patients to us. The work has been supported by grants from P. A. Brandts Legat, Christian X's Fond, Statens Almindelige Videnskabsfond, and the Fleming Memorial Fund for Medical Research.

\section{REFERENCES}

Billing, B. H., Williams, R., and Richards, T. G. (1964). Defects in hepatic transport of bilirubin in congenital hyperbilirubinaemia: an analysis of plasma bilirubin disappearance curves. Clin. Sci., 27, 245-257.

Brodersen, R. (1962). Contribution to the identification of bile pigments in normal human serum. Scand. J. clin. Lab. Invest., 14, $517-527$
(1966). Bilirubin diglucuronide in normal human blood serum. Ibid., 18, 361-379.

- Hermann, L. S., and Vind, I. (1963). Normal bilirubin concentration and the occurrence of other bile pigments in human blood sera. Ibid., 15, 523-528.

_- and Vind, I. (1963). A specific method for quantitative determination of unconjugated bilirubin and mesobilirubin. Ibid., $15,225-232$.

Overbeek, J. Th. G., Vink, C. L. J., and Deenstra, H. (1955). Kinetics of the formation of azobilirubin. Rec. Trav. chim. Pays-Bas., 74, 85-96.

Powell, W. N. (1944). A method for the quantitative determination of serum bilirubin with the photoelectric colimeter. Amer. J. clin. Path., 14, Tech. Sect. 8, 55-58.

Summerskill, W. H. (1965). The syndrome of benign recurrent cholestasis. Amer. J. Med., 38, 298-305.

Tygstrup, N. (1960). Intermittent possibly familial intrahepatic cholestatic jaundice. Lancet, 1, 1171-1172.

Williams, R., Cartter, M. A., Sherlock, S., Scheuer, P. J., and Hill, K. R. (1964). Idiopathic recurrent cholestasis: a study of the functional and pathological lesions in four cases. Quart. $J$. Med., 33, 387-399. 